\title{
Muerte Súbita Cardíaca por Fibrilación Ventricular asociada a isquemia miocárdica, tratada por Desfibrilador Automático Implantable.
}

\author{
Julián Sepúlveda ${ }^{1}$, María Lorena Jofré2 ${ }^{2}$, Gonzalo Lira ${ }^{1}$, Nicolas Veas ${ }^{3}$, Dante Lindefjeld ${ }^{3}$, José Pardo ${ }^{1}$. \\ 1. Unidad de Arritmias y Laboratorio de Electrofisiología Cardíaca. \\ 2. Unidad de Hemodinamia, Hospital Militar de Santiago. \\ 3. Unidad de Hemodinamia, Hospital Dr. Sótero del Río. \\ Conflicto de interés: ninguno. \\ Fuente de financiamiento: ninguna.
}

Reportamos el caso de un hombre de 67 años, con múltiples factores de riesgo cardiovascular, quien en el año 2015 presentó muerte súbita por fibrilación ventricular sin lesiones coronarias significativas, tras lo cual se implantó un desfibrilador automático. En el año 2019 presentó un nuevo episodio de fibrilación ventricular en relación con síndrome coronario agudo con supradesnivel del ST localizado en pared anterior, registrado y adecuadamente resuelto por el dispositivo. Se demostró una oclusión aterotrombótica en la porción proximal de la arteria descendente anterior. Fue precoz y exitosamente manejado con angioplastía coronaria percutánea e implante de stent fármaco activo, guiado por tomografía por coherencia óptica intracoronaria. A las 48 horas post angioplastía, presentó episodio de taquicardia ventricular polimorfa reconocido y tratado por el cardiodesfibrilador, sin consecuencias. Electivamente se efectuó angioplastía e implante de stent metálico en arteria coronaria derecha distal, con buen resultado angiográfico. La posterior evolución del paciente fue satisfactoria, sin manifestaciones de insuficiencia cardíaca, angina ni arritmias.

Palabras clave: Muerte súbita cardíaca; fibrilación ventricular; cardiopatía isquémica; desfibrilador automático implantable. 


\section{Sudden Cardiac Death due to Ventricular Fibrillation associated with myocardial ischemia, treated by Implantable Cardioverter Defibrillator.}

A 67-year-old man, with multiple cardiovascular risk factors who in 2015 presented sudden death due to ventricular fibrillation without significant coronary lesions, after which an implantable automatic defibrillator (ICD) was implanted. In 2019, he presented a new episode of ventricular fibrillation adequately resolved by the device, in relation to an acute coronary syndrome with ST elevation in the anterior wall of the left ventricle, due to atherothrombotic occlusion in the proximal portion of the anterior descending artery. He was early and successfully managed with percutaneous coronary angioplasty by the insertion of a drug eluting stent, implanted guided by intracoronary optical coherence tomography. Forty eight hours later, he presented an episode polymorphic ventricular tachycardia recognized and treated by the ICD. Angioplasty with a bare metal stent implantation were performed in the distal right coronary artery, with good angiographic results. The subsequent course was satisfactory, with no manifestations of heart failure, angina or arrhythmias.

Keywords: Sudden cardiac death; ventricular fibrillation; ischemic heart disease; implantable cardioverter defibrillator. 


\section{Introducción:}

Según Myerburg, la muerte súbita cardíaca (MSC) es aquella de causa cardíaca, que ocurre en forma natural, rápida e inesperada, con pérdida de conciencia dentro de la primera hora de iniciados los síntomas, en un sujeto con o sin cardiopatía preexistente. ${ }^{1}$

La MSC es la principal causa de muerte en el mundo,es responsable del $50 \%$ de las muertes cardiovasculares, afectando anualmente a 5 millones de personas al año, 700.000 en Europa y 400.000 en Estados Unidos, siendo más frecuente en hombres que en mujeres, 6.68 versus $1.4 / 100.000 .^{2}$

La cardiopatía isquémica (CI) es el substrato más frecuente de MSC, presentándose en el $80 \%$ de los casos. La incidencia de MSC asociada a CI es de 0.7/100.000 en personas entre 18-35 años y de 13.7 en mayores de $35 .{ }^{3}$ La taquicardia ventricular (TV) y la fibrilación ventricular (FV) son las responsables del colapso circulatorio y MSC en el $85 \%$ de los sujetos, en el contexto de CI, desde la isquemia aguda hasta el infarto al miocardio (IAM) ${ }^{2,4}$

El rol del desfibrilador automático implantable (DAI) es fundamental en la prevención de MSC por FV y TV, junto al control de los factores de riesgo cardiovascular y el tratamiento adecuado del síndrome coronario agudo (SCA) $, 5,6,7$

A raíz del caso comunicado, se discute el concepto de isquemia miocárdica sin lesiones coronarias obstructivas
(INOCA $^{8-9}$ : Ischemia and Non-Obstructive Coronary Artery Disease) y su eventual participación en la evolución clínica del paciente.

\section{Historia Clínica:}

Hombre de 67 años portador de hipertensión arterial, obesidad, tabaquismo antiguo, dislipidemia e insulinoresistencia, con antecedente de MSC, mientras viajaba en automóvil, el 25 de abril de 2015. Reanimado en Urgencia del Hospital Padre Hurtado, se registró FV que convirtió a ritmo sinusal (RS) con 200 Joules (J) en dos oportunidades.

Trasladado al Hospital Militar de Santiago, evolucionó sin arritmias, recuperándose sin secuela neurológica. Biomarcadores en sus valores máximos post FV: Creatinquinasa: 554 u/l (VN 38-174), CK-MB: $0.38 \mathrm{ng} / \mathrm{ml}$ (VN $<4.99)$ y Troponina I (Tn I): $1.1 \mathrm{ng} / \mathrm{l}(\mathrm{VN}<0.04)$. No se realizó curva enzimática.

El electrocardiograma (ECG) fue normal y la ecocardiografía 2D mostró motilidad conservada y fracción de eyección de ventrículo izquierdo (FEVI) normal. Al quinto día post $\mathrm{FV}$, la coronariografía mostró lesiones mínimas en arteria descendente anterior (ADA) y en arteria circunfleja $(\mathrm{ACx})$, con una lesión obstructiva de $40 \%$ en segmento medio de arteria coronaria derecha (ACD). No se dispuso de imágenes intracoronarias. Cuatro días después se instaló DAI bicameral con doble coil de desfibrilación para prevención secundaria de MSC.

\section{Figura 1.}

Secuencia angiografía de ADA en visión craneal derecha pre y post APC con implante de stent fármaco activo guiado por tomografía de coherencia óptica.
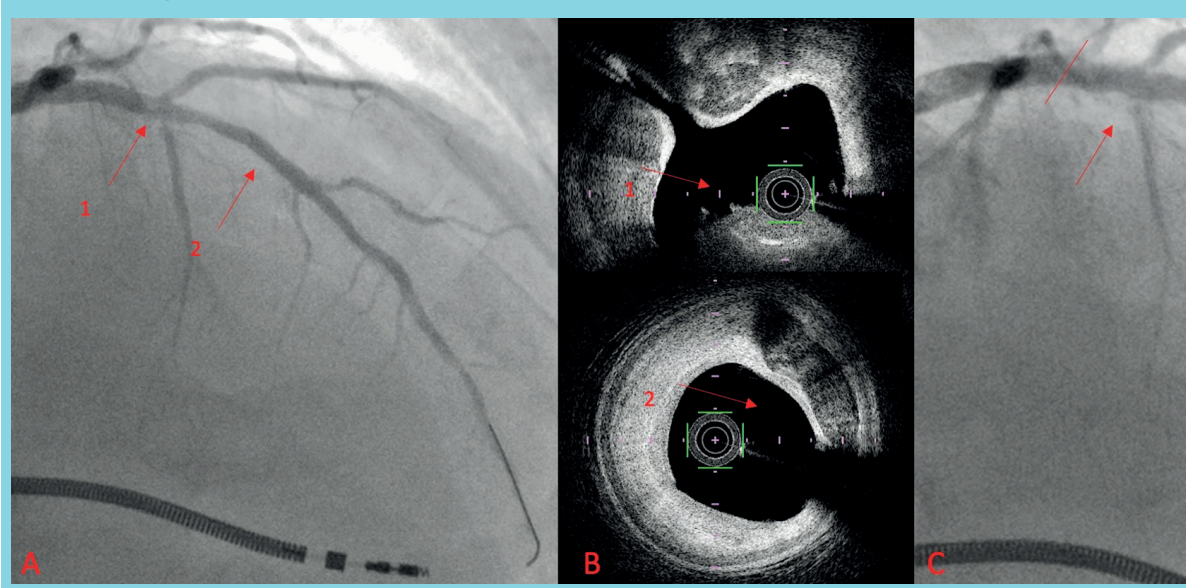

A: Angiografía coronaria de ADA en visión craneal derecha. Flecha 1: imagen radiolúcida en relación a ramo diagonal. Flecha 2: sin lesiones angiográficas significativas

B: Tomografía de coherencia óptica de ADA. 1: Correlación con flecha 1 de imagen A, la cual muestra trombo rojo sugerente de accidente de placa sobre placa fibrocálcica. 2: correlación con flecha 2 de imagen A que muestra placa fibrocálcica.

C: Angiografía de ADA en visión craneal derecha post implante de stent medicado en tercio medio. Flujo TIMI 3 final. Las marcas rojas muestran los límites del stent medicado $3.5 \times 30 \mathrm{~mm}$. 
Evolucionó asintomático bajo tratamiento con aspirina, losartán y metformina, con DAI normofuncionante y sin arritmias por telemetría, con exclusiva zona de monitoreo para $\mathrm{TV} \geq 154 \mathrm{lpm}$ y otra zona para detección $\mathrm{FV} \geq$ 214 lpm con choques de $30 \mathrm{~J}-40 \mathrm{~J}$ y $40 \mathrm{~J}$ por 6 veces.

El 9 de agosto 2019 estando en su domicilio, en reposo, presentó dolor opresivo retroesternal, sudoración profusa y fatigabilidad extrema, por lo cual es trasladado en ambulancia a Urgencia Hospital Sótero del Río, ingresando a ese Centro a los 90 minutos de iniciado el dolor, presentando en el trayecto un episodio sincopal. Con diagnóstico de SCA con elevación del ST en pared anterolateral Killip I, es sometido a coronariografía de urgencia vía arteria radial derecha sin incidentes. (Figura 1A). Destacaban arterias coronarias calcificadas, en porción media de ADA; en relación a origen de una rama diagonal presentaba una zona radiolúcida sin evidencia de lesión angiográficamente significativa, siempre con flujo adecuado a distal. Las imágenes intracoronarias con tomografía por coherencia óptica (OFDI Terumo) mostraron placas mixtas, algunas fibrolipídicas y otras fibrocálcicas. En la zona radiolúcida angiográfica se observó trombo rojo residual sugerente de accidente de placa, con un área luminal inferior a $3 \mathrm{~mm} 2$ (Figura 1B). Se efectuó angioplastía coronaria (APC) e implante de stent fármaco activo 3.5 x $30 \mathrm{~mm}$ en segmento medio de ADA (Figura 1C), con técnica de stent provisional con diagonal. Resultado satisfactorio y sin complicaciones, guiado por OFDI. La arteria coronaria derecha era dominante, ectasica y con lesión obstructiva estimada en $60 \%$. La Tn ultrasensible máxima fue 36644 ng/l (VN:45,43 ng/l).

En Unidad Coronaria (UCO), bajo tratamiento con aspirina, clopidrogel, carvedilol, atorvastatina y enalaprilo, evolucionó sin angina ni manifestaciones de insuficiencia cardíaca, observándose al ECG un hemibloqueo izquierdo anterior, QS de V1 a V3, R embrionaria V4-V5-V6 y T negativa en pared anterior de VI (Figura 3). La Eco 2D mostró hipokinesia leve en segmentos, basales, medio y apical del septum con FEVI 0.56.

A las 48 h post APC, presentó TV polimorfa convirtiendo a RS tras descarga de DAI, por lo cual se agregó amiodarona oral.

La posterior telemetría del dispositivo mostró (Figura 4) un primer episodio de TV/ FV con fecha 9 agosto 2019, a las 12:59 pm que fue precedida de extrasistolía ventricular bigeminada, convirtiendo a RS tras única descarga de $30 \mathrm{~J}$, coincidiendo con síncope presentado en ambulancia. La segunda TV polimorfa registrada y resuelta satisfactoriamente por DAI, correspondió a episodio presentado en UCO. La batería de DAI, parámetros de sensado, estimulación e impedancia se encontraban en límites normales.

Hasta la fecha el paciente se mantiene asintomático coronario sin cambios en su tratamiento farmacológico post SCA, en capacidad funcional I y sin arritmias detectadas en DAI.

\section{Discusión:}

La MSC es el resultado final de una cadena de eventos que derivan en FV y paro cardiorespiratorio (PCR) produciendo una contracción irregular y caótica del miocardio ventricular. ${ }^{2,4}$

La supervivencia tras PCR extrahospitalario es menor al $10 \%$, de acuerdo a distintos registros, lo que dimensiona el impacto del manejo oportuno y su prevención. ${ }^{10,11}$ El miocardio queda vulnerable por isquemia y/o altera-

\section{Figura 2.}

Secuencia angiografía de $A C D$ en visión craneal izquierda pre y post APC con implante de stent metálico. En ventrículo derecho se observa coil de cable ventricular de DAI.

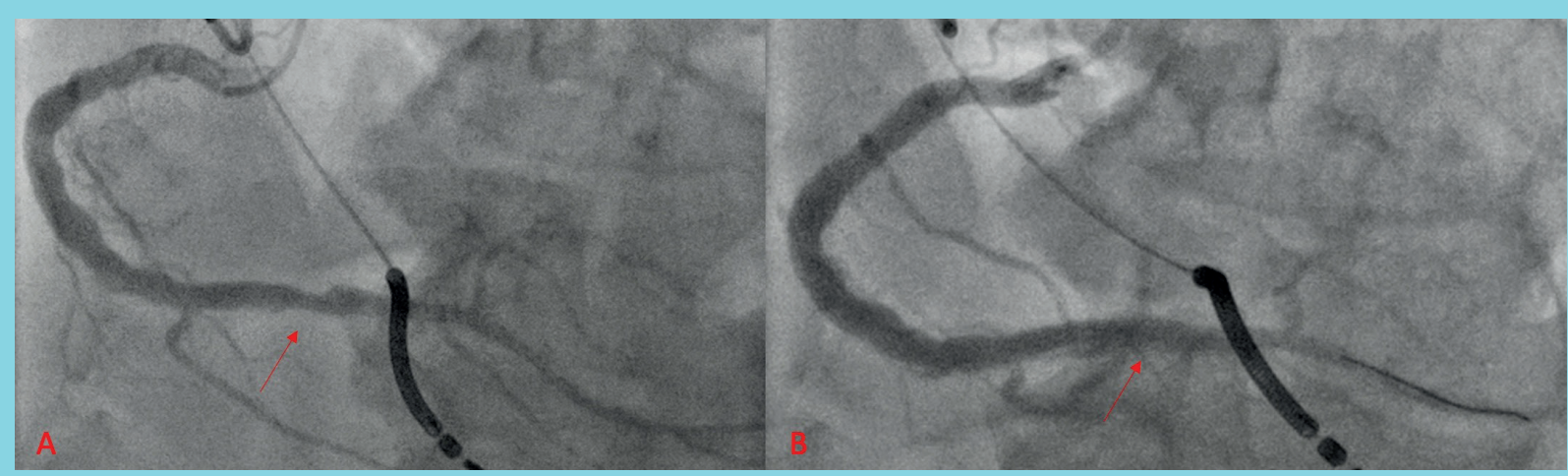

A: Angiografía de ACD dominante en visión craneal izquierda con lesión de $70 \%$ en tercio distal (flecha).

B: Resultado post APC e implante de stent (flecha). Flujo Timi 3 final. 
Figura 3.

ECG 11 agosto 2019

Ritmo sinusal, hemibloqueo izquierdo anterior, QS de V1 a V3, con R embrionaria de V4 a V6, y T negativa en pared anterior de ventrículo izquierdo.

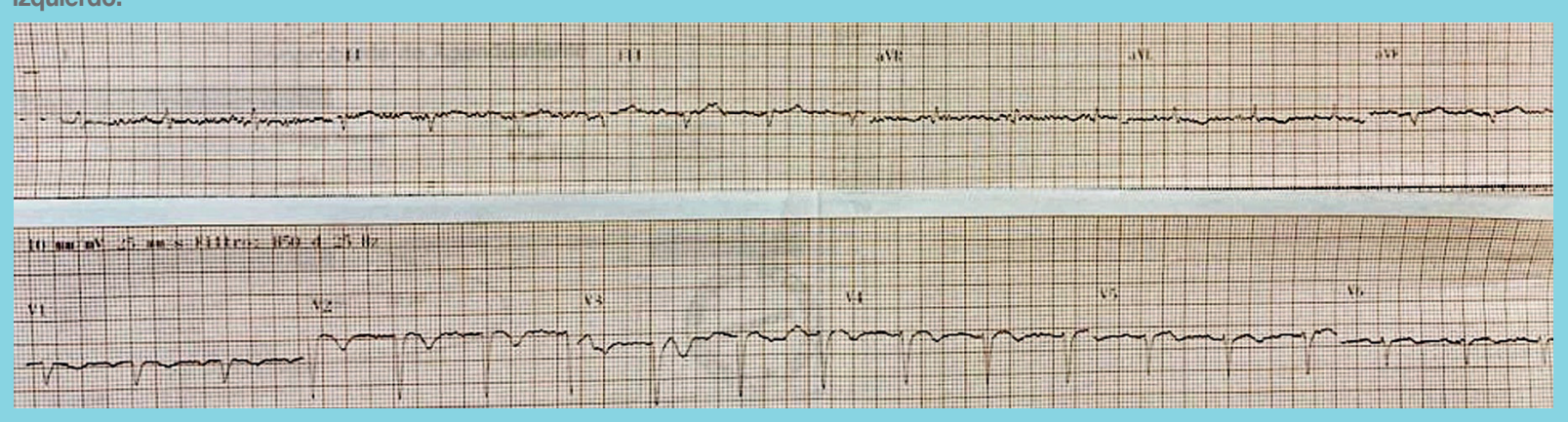

ciones estructurales, en un ambiente inestable por fluctuaciones en el tono autonómico, nivel de catecolaminas circulantes, alteraciones hemodinámicas, hidroelectrolí- ticas, tóxicos o fármacos, condiciones ideales para arritmias ventriculares. ${ }^{12,13}$.

Los mecanismos subyacentes en FV, incluyen circuitos

Figura 4.

Secuencia cronológica de electrogramas intracavitarios registrados por DAl, en canal de marca eléctrica (AV), canal de campo lejano (CL), canal auricular (A) y canal ventricular ( $\mathrm{V}$ ).
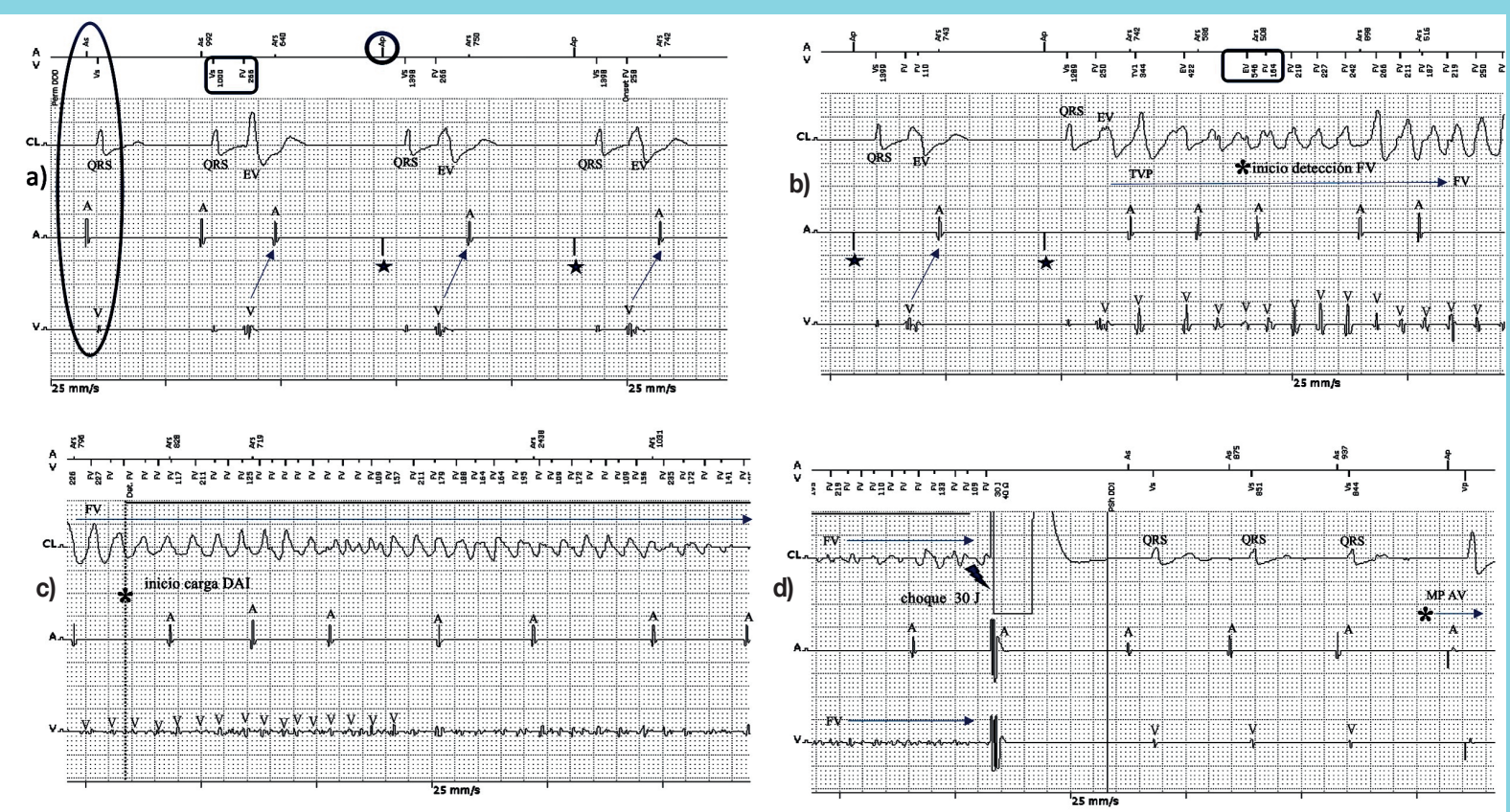

a) En el primer complejo QRS en canal CL (elipse) se reconoce actividad sinusal intracavitaria con señal auricular (A), seguida de señal ventricular (V), con adecuado sensado de marca eléctrica en canal AV (AsVs). En canal AV se observa que primer extrasistole ventricular (EV) es sensado a 266 mseg del complejo QRS cayendo en zona de detección de FV (rectángulo) de acuerdo a programación DAI. A continuación, se observa EV bigeminada con conducción retrógrada (flecha) registrada en canal $V$ y A respectivamente. Tras primer EV se observa marca de espiga auricular en canal AV (Ap en circulo) pero sin captura en canal A (estrella).

b) En el canal de CL tras el segundo complejo QRS, un EV da origen a una TV polimorfa observándose disociación AV en canal A y V, donde la señal $V$ es amplia y organizada. Progresivamente se desorganiza como FV, la que es adecuadamente reconocida en canal AV (rectángulo) dando inicio a la detección y conteo de complejos QRS (asterisco) previo al choque de 30J.

c) En el canal CL se observa persistencia de FV con disociación AV en canal A y V, con desorganización y disminución significativa de señal V. En (asterisco) el inicio de carga de DAI, calculado en 6.8 s, para entregar choque de $30 \mathrm{~J}$.

d) En canal CL se observa el momento de choque de $30 \mathrm{~J}$ (flecha) sobre $\mathrm{FV}$, restaurando de inmediato el ritmo sinusal con registro de tres primeros complejos QRS con secuencia normal de señal A y V en respectivos canales. El último complejo QRS se genera por estimulación bicameral con señal Ap y Vp en canal AV (asterisco). 
de reentrada en áreas isquémico-necróticas, automatismo anormal y actividad desencadenada por dispersión de períodos refractarios. ${ }^{12}$

La única estrategia que ha mostrado ser efectiva en prevención primaria como secundaria de MSC por FV es el DAI. ${ }^{6,7}$

El DAI termina el episodio de arritmia ventricular, sin alterar su mecanismo subyacente. Por lo tanto, el tratamiento además implica resolver los factores precipitantes, el uso de antiarrítmicos y eventualmente terapias de ablación. ${ }^{6,10,12}$

Nuestro paciente tras recuperación de primera MSC por FV en año 2015, recibió DAI como prevención secundaria constituyendo una indicación clase I, nivel de evidencia $A^{6}$.

El primer evento de FV del año 2015, ocurrió en presencia de lesiones coronarias no significativas, pero de muy probable etiología isquémica aguda que no se pudo confirmar por falta de imágenes intracoronarias.

Recientemente, se ha utilizado el término INOCA, para describir aquellos pacientes que se presentan con isquemia miocárdica sin obstrucción coronaria significativa ( $<50 \%$ de estenosis por angiografía), atribuida a disfunción coronaria microvascular, vasoespasmo coronario o la combinación de ambos, manifestándose con angina de reposo hasta IAM $^{8,9}$

Más frecuente en mujeres (65\%), sus factores de riesgo son la obesidad, dislipidemia, diabetes, hipertensión y tabaquismo. 8,9

En la fisiopatología de INOCA a nivel microvascular, se presentan alteraciones estructurales como remodelación, rarefacción arteriolar y funcionales, con disminución de biodisponibilidad de óxido nítrico y aumento de endotelina-1, cuyo resultado final es la hipoperfusión e isquemia. ${ }^{8-9}$

La disfunción microvascular coincide con etapas tempranas de aterosclerosis, antes de cualquier evidencia angiográfica de obstrucción coronaria, al permitir el paso de material lipídico al espacio subintimal, donde una potente respuesta inflamatoria mediada, principalmente, por macrófagos, permite una acumulación progresiva de cristales de colesterol y restos celulares, que perpetúan la inflamación y progresión de la placa. ${ }^{15}$

Enfrentar un caso de INOCA, implica un gran desafío diagnóstico, al requerir estudios adicionales para evaluar flujo y reserva coronaria, a través de angiografía por tomografía axial computarizada, ecocardiografía doppler, cardioresonancia magnética (CRM), tomografía por emisión de positrones, coronariografía y prueba farmacológica, cuyo análisis va más allá de los objetivos de este manuscrito. 8,9

La CRM actualmente, como método no invasivo, juega un rol fundamental en la caracterización tisular y evaluación de los defectos de perfusión por estenosis de vasos epicardicos o por disfunción microvascular. ${ }^{8,9}$

Cuatro años después el paciente se presentó con nuevo episodio de FV secundario a IAM tipo I según la cuarta definición universal ${ }^{7}$, confirmándose por OFDI la presencia de un trombo fresco oclusivo con accidente de placa como causa de isquemia aguda.

No hemos encontrado en la literatura una clara relación entre INOCA y FV, pero dada la presentación inicial y posterior evolución clínica de la CI de nuestro paciente, no podemos dejar de considerar esta nueva entidad, considerada de mal pronóstico, muchas veces subdiagnosticada y subtratada, como mecanismo responsable del primer episodio de MSC. ${ }^{8,9}$ 


\section{Referencias}

1. MYERBURG J, CASTELLANOS A. (1992) CARDIAC ARREST AND SUDDEN CARDIAC DEATH. BRAUNWALD, E. HEART DISEASE. A Textbook of Cardiovascular Medicine. (Fourth Edition 756-789).W.B Saunders Company.

2. SZABÓ Z, UJVÁROSY D, ÖTVÖS T, SEBESTYÉN V, NÁNÁSI PP. Handling of Ventricular Fibrillation in the Emergency Setting. Front Pharmacol. 2020;10:1640. Published 2020 Jan 29. doi:10.3389/fphar.2019.01640

3. KATRITSIS DG, GERSH BJ, CAMM AJ. A Clinical Perspective on Sudden Cardiac Death. Arrhythm Electrophysiol Rev. 2016;5(3):177-182. doi:10.15420/aer.2016:11:2

4. JAZAYERI MA, EMERT MP. Sudden Cardiac Death: Who Is at Risk? The Medical Clinics of North America. 2019 Sep;103(5):913-930. DOI: 10.1016/j.mena.2019.04.006.

5. MORIN DP, HOMOUD MK, Estes NAM 3rd. Prediction and Prevention of Sudden Cardiac Death. Card Electrophysiol Clin. 2017;9(4):631-638. doi:10.1016/j.ccep.2017.07.012.

6. AL-KHATIB SM, STEVENSON WG, ACKERMAN MJ, BRYANT WJ, CALLANS DJ, CURTIS AB et al. 2017 AHA/ ACC/HRS guideline for management of patients with ventricular arrhythmias and the prevention of sudden cardiac death: Executive summary: A Report of the American College of Cardiology/American Heart Association Task Force on Clinical Practice Guidelines and the Heart Rhythm Society. Heart Rhythm. 2018;15(10): e190-e252. doi:10.1016/j.hrthm.2017.10.035.

7. THYGESEN K, ALPERT JS, JAFFE AS, CHAITMAN BR, BAX JJ, MORROW DA et al. Fourth universal definition of myocardial infarction (2018) [Fourth universal definition of myocardial infarction (2018)]. Kardiol Pol. 2018; 76 (10):1383-1415. doi:10.5603/KP.2018.0203.

8. KUNADIAN V, CHIEFFO A, CAMICI PG, et al. An EAPCI
Expert Consensus Document on Ischaemia with Non-Obstructive Coronary Arteries in Collaboration with European Society of Cardiology Working Group on Coronary Pathophysiology \& Microcirculation Endorsed by Coronary Vasomotor Disorders International Study Group [published online ahead of print, 2020 Jul 6]. EuroIntervention. 2020;EIJY20M07_01. doi:10.4244/EIJY20M07_01

9. PADRO T, MANFRINI O, BUGIARDINI R, et al. ESC Working Group on Coronary Pathophysiology and Microcirculation position paper on 'coronary microvascular dysfunction in cardiovascular disease'. Cardiovasc Res. 2020;116(4):741755. doi:10.1093/cvr/cvaa003

10. BHAR-AMATO J, DAVIES W, AGARWAL S. Ventricular Arrhythmia after Acute Myocardial Infarction: 'The Perfect Storm'. Arrhythm Electrophysiol Rev. 2017;6(3):134-139. doi:10.15420/aer.2017.24.

11. GIROTRA S, VAN DIEPEN S, NALLAMOTHU BK, et al. Regional Variation in Out-of-Hospital Cardiac Arrest Survival in the United States. Circulation. 2016;133(22):21592168. doi:10.1161/CIRCULATIONAHA.115.018175

12. GOYAL V, JASSAL DS, DHALLA NS. Pathophysiology and prevention of sudden cardiac death. Can J Physiol Pharmacol. 2016;94(3):237-244. doi:10.1139/cjpp-2015-0366

13. NICHOL G, SAYRE MR, GUERRA F, POOLE J. Defibrillation for Ventricular Fibrillation: A Shocking Update. J Am Coll Cardiol. 2017;70(12):1496-1509. doi:10.1016/j. jacc.2017.07.778

14. BELTRAME J. Assessing patients with myocardial infarction and nonobstructed coronary arteries (MINOCA). J Intern Med. 2013; 273(2):182-5. doi: 10.1111/j.1365-2796.2012.02591.x

15. LANZA G, CREA F. Primary coronary microvascular dysfunction: clinical presentation, pathophysiology, and management. Circulation 2010; 121(21):2317-25. doi: 10.1161/ CIRCULATIONAHA.109.900191 